\title{
LCC from December 2018-February 2019 Lists
}

Compiled by Ann Heinrichs

The new classification numbers listed here reflect the most recent information available at the time of publication. Items in this list were selected from the 2018 list number 12 (December 17) and the 2019 list numbers 01 (January 21) and 02 (February 18).

\section{CLASS B}

B3149.E8

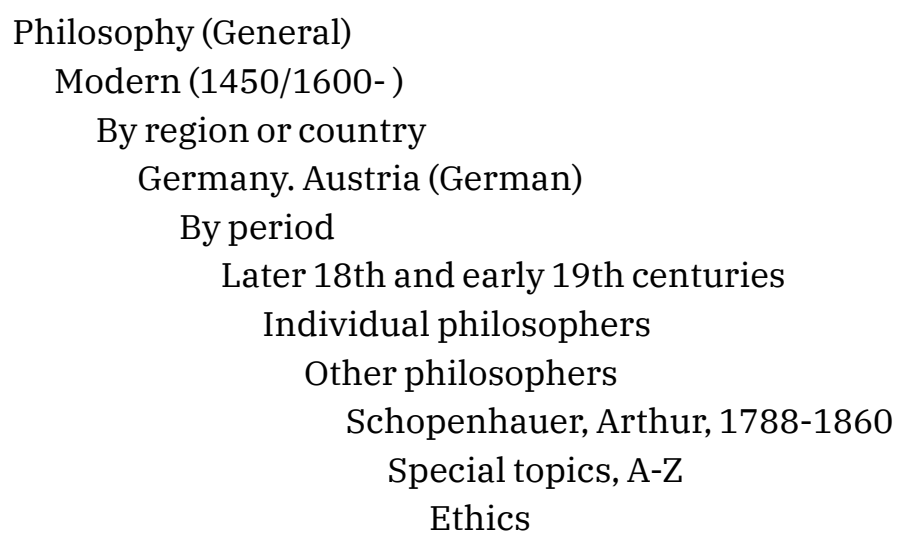

\section{CLASS BL}

BL65.B73

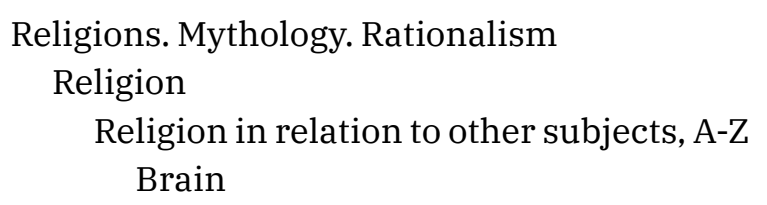

[BL1279.89-.8922]

Biography CANCEL LINE 
BL1279.89

BL1279.892.A-Z

BL1279.892.D37

BL1288.292.M83
Collective CANCEL LINE

Founders and most important leaders, A-Z

CANCEL LINE

Dāsa, Rāma Ratana, 1908-1964 TABLE BL4

CANCEL LINE

Hinduism

Modifications. Sects. Movements. Cults

Individual sects, movements, cults

Vaishnavism

Srivaisnavas (Ramanuja sect)

Biography

Founders and most important leaders, A-Z

Mudaliyāṇ̣̂̄n, 1027-1132 TABLE BL4

By region or country

Southeast Asia

By region or country

Vietnam

By ethnic group, A-Z

BL2059.T37

Tày (Northern Vietnamese people)

CLASS BM

BM496.9.P76

Judaism

Sources

Rabbinical literature

Special topics, A-Z

BM496.9.W58

Prostitutes

Witches

Practical Judaism

BM685.4

Melavveh malkah. Meal of King David. הכלמ הוולמ

BM728.5 Public theology

CLASS BP

BP190.5.M256

Islam. Bahai Faith. Theosophy, etc.

Islam

Topics (not otherwise provided for), A-Z Man-woman relationships 
10 THEOLOGY CATALOGING BULLETIN • APRIL 2019: VOL. 27, NO 2

\title{
CLASS BR
}

\author{
Christianity \\ Collected works \\ Early Christian literature to ca. 600. Fathers of the Church, etc. \\ Individual authors
}

BR65.A41-.A416 Arnobius, of Sicca TABLE BR1

BR65.H7-.H76 Hilary, Saint, Bishop of Poitiers, -367 TABLE BR1

BR1720.P254

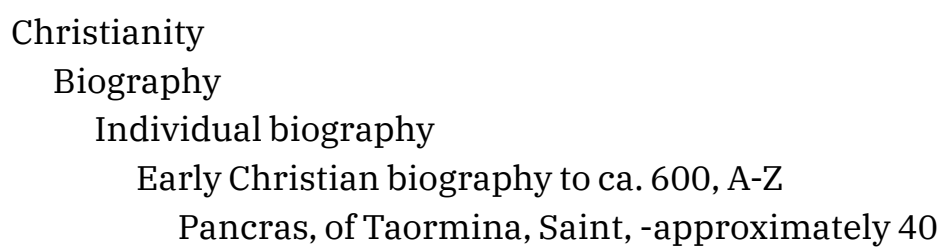

\section{CLASS BS}

BS325.M24

The Bible

General

Texts and versions

Modern texts and versions

Non-European languages

African languages, $\mathrm{A}-\mathrm{Z}$

Ma'di TABLE BS5

\section{CLASS BT}

BT580.G83

\section{Doctrinal theology}

Christology

Miracles. Apparitions. Shrines, sanctuaries, images, processions, etc. Special. By place, A-Z

$$
\text { Guatemala (Guatemala) }
$$

\section{CLASS BX}

BX4089

\author{
Christian denominations \\ Catholic Church \\ Monasticism. Religious orders \\ Individual orders of men \\ Towarzystwo Chrystusowe dla Polonii Zagranicznej TABLE BX18
}


BX4705.K6957

BX7062

CLASS DR

DR435.K36

CLASS KBP

KBP320.S63

\section{CLASS LC}

LC495.S25

\author{
Catholic Church \\ Biography and portraits \\ Individual \\ Other, A-Z \\ Krebs, Engelbert, 1881-1950
Christian denominations
Other Protestant denominations
Church of God of Union Assembly TABLE BX3

History of Balkan Peninsula

Turkey

Ethnography

Individual elements in the population, $\mathrm{A}-\mathrm{Z}$ Karamanlides

تقف .قعيرش. Islamic law. Sharī ‘ ah. Fiqh

Schools of thought. Islamic legal schools. Madhāhib. بهاذم

Schools and authors affiliated with a particular school

Sunnī schools

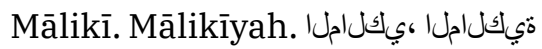

Individual authors, A-Z

Sijilmāsī, Ibrāhīm ibn Hilāl, 1414 or 1415-1497 or 1498. لالنب مي:اربا، ،يساجلجس TABLE K4

Special aspects of education

Moral and religious education

Religion and education. Education under church control

Christian education. Church education

Roman Catholic

Religious orders

Other special, A-Z

Salesian 


\section{CLASS N}

Visual arts
Special subjects of art
Other special subjects (alphabetically)
A - Industry
N8217.C793 Crusades

\section{CLASS NC}

NC1763.I48

Drawing. Design. Illustration

Caricature. Pictorial humor and satire

Special subjects, A-Z

Inquisition

\section{CLASS ND}

ND3359.B524

Painting

Illuminating of manuscripts and books

Histories and reproductions of special illuminated works

Special works

Bibles

New Testaments

Evangelaries, A-Z

Bibliothèque de l’Arsenal. Manuscript. 592

\section{CLASS PG}

PG3415.S65

Slavic. Baltic. Albanian

Russian literature

Individual authors and works, $1800-1870$

Tolsto1̆, Lev Nikolaevich, graf, 1828-1910 (Tolstoy, Leo). Лев Николаевич Толстой Biography and criticism Criticism and interpretation

Treatment and knowledge of special subjects, A-Z Spirituality

\section{CLASS PL}

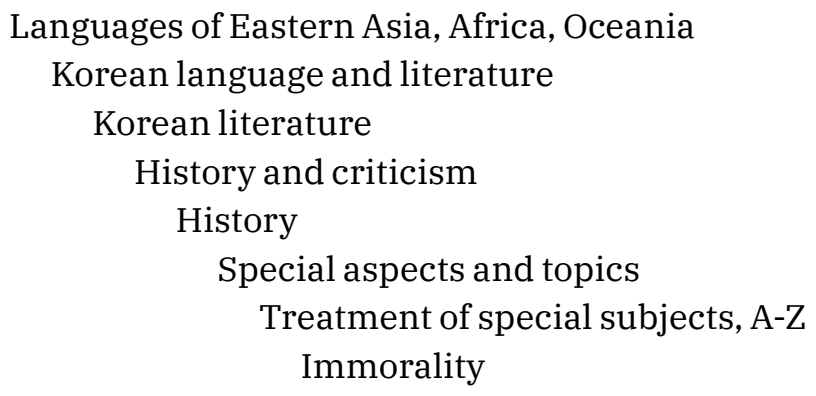




\section{CLASS PR}

PR153.J63

English literature

History of English literature

Special topics not limited to, or identified with, one period or form Special characters, A-Z

Joan, of Arc, Saint, 1412-1431

\section{CLASS Z}

[Z667.6-.63]

(Z667.6-.63)

Z667.6

Z667.63

Libraries

Library science. Information science Information storage and retrieval systems Information filtering systems CANCEL

Information filtering systems - see ZA3083-3085

General works CANCEL LINE

Recommender systems CANCEL LINE

Personnel

Special groups, A-Z

Z682.4.D38 Data services librarians

Z682.4.E46 Embedded librarians

\section{CLASS ZA}

[ZA3083-3085]

Information resources (General)

Research. Seeking and finding information. Information retrieval

ZA3083

General works

ZA3084

Recommender systems

ZA3085

Filter bubbles 